\title{
Temporal trend in the natural history of ulcerative colitis in a country with a low incidence of ulcerative colitis from 2000 through 2018
}

\author{
Satimai Aniwan ${ }^{1,2}$, Julajak Limsrivilai ${ }^{3}$, Supot Pongprasobchai ${ }^{3}$, Nonthalee Pausawasdi ${ }^{3}$, Piyapan Prueksapanich ${ }^{1,2}$, \\ Natanong Kongtub ${ }^{1,2}$, Rungsun Rerknimitr ${ }^{1,2}$ \\ ${ }^{1}$ Division of Gastroenterology, Faculty of Medicine, Chulalongkorn University, Bangkok; ${ }^{2}$ King Chulalongkorn Memorial Hospital, The Thai \\ Red Cross Society, Bangkok; ${ }^{3}$ Division of Gastroenterology, Department of Internal Medicine, Faculty of Medicine, Siriraj Hospital, Bangkok, \\ Thailand
}

Background/Aims: The incidence of ulcerative colitis (UC) in Thailand (crude incidence rate of 0.28 per 100,000 persons) is much lower than in the West. The burden of UC varies in different populations. The aim of this study was to evaluate the natural history of UC over the two decades in Bangkok, Thailand. Methods: This retrospective study included patients who were diagnosed with UC between 2000 and 2018 in 2 university hospitals. To evaluate changes in the disease course, we stratified patients into 2000-2009 cohort and 2010-2018 cohort. The cumulative probability of endoscopic healing, UC-related hospitalization and colectomy was estimated using the Kaplan-Meier method. Results: A total of 291 UC patients were followed for total of 2,228 person-years. Comparison between 2 cohorts, there were no differences in disease pattern and severity whereas an increase in the combination use of oral and topical mesalamine and the early use of thiopurine was observed. Only $1 \%$ of patients for each cohort required biologic agent at 5 years. The rate of achieving mucosal healing increased from $15 \%$ to $46 \%$ at 3 years $(P<0.01)$. The rate of UC-related hospitalization decreased from $30 \%$ to $21 \%$ at 5 years $(P<0.05)$. The rate of colectomy decreased from $6 \%$ to $2 \%$ at 5 years $(P<0.05)$. Conclusions: The natural history of UC in a low incidence country was less aggressive than the West. Over the past two decades, the rates of UC-related hospitalization and colectomy have been decreasing which were similar to the West. (Intest Res 2021;19:186-193)

Key Words: Colitis, ulcerative; Inflammatory bowel disease; Epidemiology; Natural history

\section{INTRODUCTION}

Ulcerative colitis (UC) is an incurable chronic disease that commonly develops at young ages and lasts throughout life, impacting quality of life. Although a recent systematic review showed that more than $80 \%$ of studies from high prevalence regions such as North America and Western Europe reported a plateau or decreased incidence of UC, the prevalence of UC remains

Received February 17, 2020. Revised April 25, 2020. Accepted May 23, 2020. Correspondence to Satimai Aniwan, Division of Gastroenterology, Department of Internal Medicine, Faculty of Medicine, Chulalongkorn University, 1873 Rama IV Road Pattumwan, Bangkok 10330, Thailand. Tel: +66-2-256-4265, Fax: +66-2-252-7839, E-mail: satimai@gmail.com high. In contrast, a low prevalence region, such as Asia, which has newly industrialized countries, is facing increasing incidence. ${ }^{1}$ Therefore, changing the epidemiology of UC in the next decade will challenge health care systems worldwide.

The burden of UC varies in different countries and populations. Although UC is not associated with an increased risk of mortality, it is associated with high morbidity. ${ }^{3}$ Because of the complex and incurable nature of the disease, understanding the natural history of UC is important to prepare health care resources and policies for this chronic and costly disease. A systematic review of the natural history of UC that included several studies from high incidence countries reported that approximately $50 \%$ of patients with UC required corticoste- 
roids at 10 years, the rate of UC-related hospitalization at 10 years was $50 \%$, and the rate of colectomy at 10 years was $10 \%$ to $15 \% .{ }^{3}$ However, long-term studies from low incidence countries are lacking. In Asia-Pacific region, Thailand had the lowest incidence of UC (crude incidence of 0.28 per 100,000 persons). ${ }^{4}$

Hence, we aimed to evaluate the natural history of adult UC in a low incidence area and changes of disease course regarding the rates of endoscopic healing, hospitalization and colectomy between 2000 and 2018.

\section{METHODS}

\section{Study Setting and Study Population}

We conducted a retrospective study in 2 university hospitals (Siriraj Hospital and King Chulalongkorn Memorial Hospital) from Bangkok, Thailand. All patients were required to meet the diagnosis criteria of UC on the basis of clinical symptoms, diffusely granular or friable colonic mucosa, and continuous mucosal involvement based on endoscopy and histology., We excluded UC patients who were diagnosed from outside our institutes. Cases were included only for the remaining UC diagnosis at 6 months after the first date of diagnosis. Infections, intestinal tuberculosis, amoebiasis, and drug-induced colitis were excluded. The index date was based on the first UC diagnosis. The medical records of patients with UC were retrospectively reviewed from the date of first UC diagnosis until the end of the study (December 31, 2018) by 2 experts (S.A. and J.L.). The study protocol was approved by the ethics committees of each institute (Chulalongkorn IRB No. 398/53 and Siriraj IRB No. 710/2561). The informed consent was waived.

\section{Treatment Strategy}

In our practice, the treatment strategy is based on a step-up approach. For mild to moderate disease activity, mesalamine (topical and/or oral) is prescribed as induction and maintenance. For severe disease activity, systemic corticosteroids are prescribed. Thiopurine is prescribed for steroid-dependent UC. Biologic agents are prescribed for steroid refractory or steroid dependent with thiopurine nonresponsive/intolerant. During the study period, infliximab and vedolizumab are available for UC indication in Thailand. According to the Food and Drug Administration of Thailand, infliximab has been approved since 2003 and vedolizumab has been approved since 2017. Infliximab was able to be reimbursed since 2009. The criteria for reimbursement of infliximab are steroid refractory or steroid dependent with thiopurine nonresponsive/intolerant. Nowadays, vedolizumab is non-reimbursable. Colectomy is considered for severe UC refractory to medical treatment. Colectomy in UC patients is only performed at our institutes. Patients with induction therapy are followed up every 1-2 months and patients with maintenance therapy are followed up every 3-6 months at the inflammatory bowel disease clinic in our institutes. Endoscopic assessment is performed within a 6- to 12-month interval after treatment initiation.

\section{Data Collection}

At UC diagnosis, data on demographic characteristics, smoking status, symptom duration before diagnosis, and disease extent were collected. During the follow-up period, data on endoscopic healing, UC-related hospitalization and colectomy were collected. Data regarding UC treatment was collected as follows: (1) ever used oral mesalamine; (2) ever used combined topical and oral mesalamine; (3) ever used systemic corticosteroid; (4) ever used thiopurine and date of initiation; (5) ever used biologic agent and date of initiation; (6) at least one hospital admission more than 24 hours and date of first admission; and (7) colectomy and date of first colonic resection. We defined requiring systemic corticosteroid, biologic agent, and colectomy due to medical failure as markers of disease severity. The extent of disease was defined according to the Montreal classification as proctitis (E1), left-sided colitis (E2) and extensive colitis (E3). We defined mucosal healing as Mayo endoscopic subscores (0-1).

\section{Statistical Analysis}

The chi-square or Fisher exact test was used for 2 proportions of data comparison. Independent $t$-tests or the Mann-Whitney $U$ test was used for 2 continuous data comparison. To assess changes in the disease course of UC, we stratified patients into 2 cohorts: (1) 2000-2009 cohort-patients with UC diagnosis during the calendar year of 2000-2009 and (2) 2010-2018 cohort-patients with UC diagnosis during the calendar year of 2010-2018. The cumulative probability of endoscopic healing, UC-related hospitalization and colectomy was estimated using the Kaplan-Meier method. The log-rank test was used to compare the disease course of UC between the 2 cohorts. Cox proportional hazards analyses were used to assess association between baseline factors (sex, age $<40$ years at diagnosis, body mass index, smoker, extensive colitis, extraintestinal manifestation and calendar period of UC diagnosis) and time from UC diagnosis to first event of achieving endoscopic healing, 
UC-related hospitalization, and colectomy expressed in hazard ratio (HR) with 95\% confidence interval (CI). We included variables with a $P<0.1$ in the univariate analysis in the multivariate analysis model. We considered statistically significant as an alpha level of 0.05 . Statistical analyses were performed by using SPSS Statistics version 23.0 (IBM Corp., Armonk, NY, USA).

\section{RESULTS}

\section{Patient Characteristics}

A total of 291 patients with UC (119 patients diagnosed in 2000-2009 and 172 patients diagnosed in 2010-2018) were followed for total of 2,228 person-years. The median follow-up duration in 2000-2009 cohort was 10 years (interquartile range [IQR], 7-13 years) and 4 years (IQR, 2-7 years) in 2010-
2018 cohort. The median age at UC diagnosis was 42 years (IQR, 32-55 years), and 53\% of patients were female. There were nonsmokers ( $\mathrm{n}=261,90 \%)$, former smokers $(\mathrm{n}=27,9 \%)$ and active smokers $(\mathrm{n}=3,1 \%)$. Twelve patients $(4 \%)$ underwent appendectomy. None of the patients had a family history of inflammatory bowel disease. At diagnosis, the disease extent was distributed as E1 $(\mathrm{n}=62,21 \%), \mathrm{E} 2(\mathrm{n}=103,35 \%)$, and E3 $(\mathrm{n}=126,43 \%)$. During study period, 23 (14\%) patients with either E1or E2 at diagnosis had disease progression to E3. Eightyone (68\%) patients in the 2000-2009 cohort and 112 (65\%) patients in the 2010-2018 cohort experienced severe disease $(P=0.60)$. There was no difference in patient characteristics between 2 cohorts (Table 1).

\section{Medications}

Comparing between the 2 cohorts, the use of combination

Table 1. Characteristics of 291 Patients with UC Diagnosed between 2000-2009 and 2010-2018

\begin{tabular}{|c|c|c|c|}
\hline \multirow{2}{*}{ Characteristics } & \multicolumn{2}{|c|}{ UC cohort $(n=291)$} & \multirow{2}{*}{$P$-value } \\
\hline & $2000-2009(n=119)$ & $2010-2018(n=172)$ & \\
\hline Female sex & $63(53)$ & $88(51)$ & 0.77 \\
\hline Median age at diagnosis (yr) & $42(32-52)$ & $42(32-58)$ & 0.16 \\
\hline Median duration of symptom onset (yr) & $0.4(0.2-1.1)$ & $0.4(0.1-1.0)$ & 0.99 \\
\hline $\mathrm{BMI}$ at diagnosis $\left(\mathrm{kg} / \mathrm{m}^{2}\right)$ & $22.1 \pm 3.7$ & $21.9 \pm 3.4$ & 0.68 \\
\hline Smoking status at diagnosis & & & 0.90 \\
\hline Current smoker & $1(1)$ & $2(1)$ & \\
\hline Ex-smoker & $12(10)$ & $15(9)$ & \\
\hline Non-smoker & 106 (89) & $155(90)$ & \\
\hline Appendectomy & $6(5)$ & $6(4)$ & 0.51 \\
\hline First degree relative with IBD diagnosis & 0 & 0 & NA \\
\hline UC location at diagnosis & & & 0.75 \\
\hline Proctitis & $23(20)$ & $39(23)$ & \\
\hline Left-sided colitis & $42(35)$ & $61(35)$ & \\
\hline Extensive colitis & $54(45)$ & $72(42)$ & \\
\hline Extraintestinal manifestation & $13(10)$ & $16(9)$ & 0.65 \\
\hline \multicolumn{4}{|l|}{ Ever used UC medications } \\
\hline Oral mesalamine & $85(71)$ & $132(77)$ & 0.31 \\
\hline Combined topical and oral mesalamine & $34(29)$ & $85(49)$ & $<0.01$ \\
\hline Systemic corticosteroids & $75(63)$ & $110(64)$ & 0.87 \\
\hline Thiopurine & $65(55)$ & $90(52)$ & 0.70 \\
\hline Biologic agent & $3(3)$ & $1(1)$ & 0.31 \\
\hline UC disease severity ${ }^{\mathrm{a}}$ & $81(68)$ & $112(65)$ & 0.60 \\
\hline
\end{tabular}

Values are presented as number (\%), median (interquartile range), or mean \pm standard deviation.

${ }^{a} U C$ disease severity defined as requiring systemic corticosteroid, biologic agent, or colectomy.

UC, ulcerative colitis; BMI, body mass index; IBD, inflammatory bowel disease; NA, not available. 
treatment of oral and topical mesalamine in the 2010-2018 cohort (49\%) significantly increased when compared with the use in the $2000-2009$ cohort $(29 \%, P<0.05)$. For systemic corticosteroids, there was no difference in any use of corticosteroids between the 2 cohorts ( $63 \%$ vs. $64 \%, P=0.87$ ). For thiopurine use, a significant increase in the use of thiopurine between the 2 cohorts was observed. The median duration from diagnosis to thiopurine initiation in the 2000-2009 cohort was 24 months and 11 months in the 2010-2018 cohort $(P<0.01)$. The cumulative probability of receiving thiopurine was $21 \%$ versus $29 \%$ at 1 year and $35 \%$ versus $49 \%$ at 3 years and $42 \%$ versus $63 \%$ at 5 years after diagnosis between the 2000-2009

A

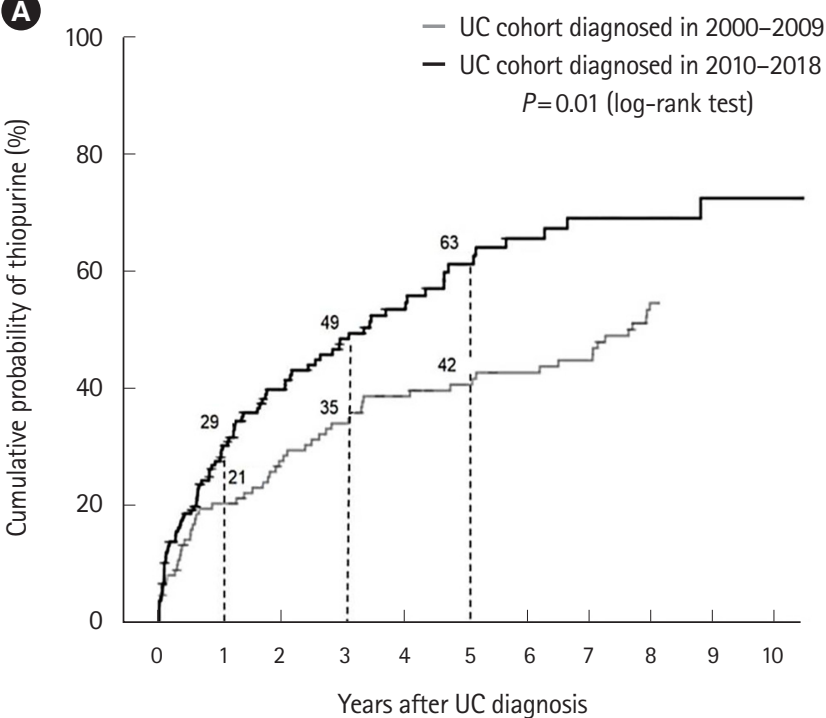

No. at risk

$\begin{array}{llllllllllll}\text { 2000-2009 cohort } & 119 & 89 & 78 & 71 & 62 & 57 & 54 & 51 & 39 & 36 & 31\end{array}$

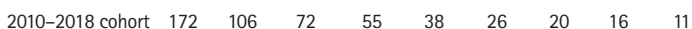

cohort and the 2010-2018 cohort $(P=0.01)$ (Fig. 1A). Only 3 patients in the 2000-2009 cohort and 1 patient in the 20102018 cohort required biologic agents. The cumulative probability of receiving biologic agents was $1 \%$ at 5 years after diagnosis for both cohorts.

\section{Endoscopic Healing}

A total of 252 patients with UC underwent endoscopic evaluation after treatment. There was a significant increase in the mucosal healing rate between the 2 cohorts. The cumulative probability of achieving mucosal healing was $8 \%$ versus $16 \%$ at 1 year, $15 \%$ versus $46 \%$ at 3 years and $27 \%$ versus $58 \%$ at 5

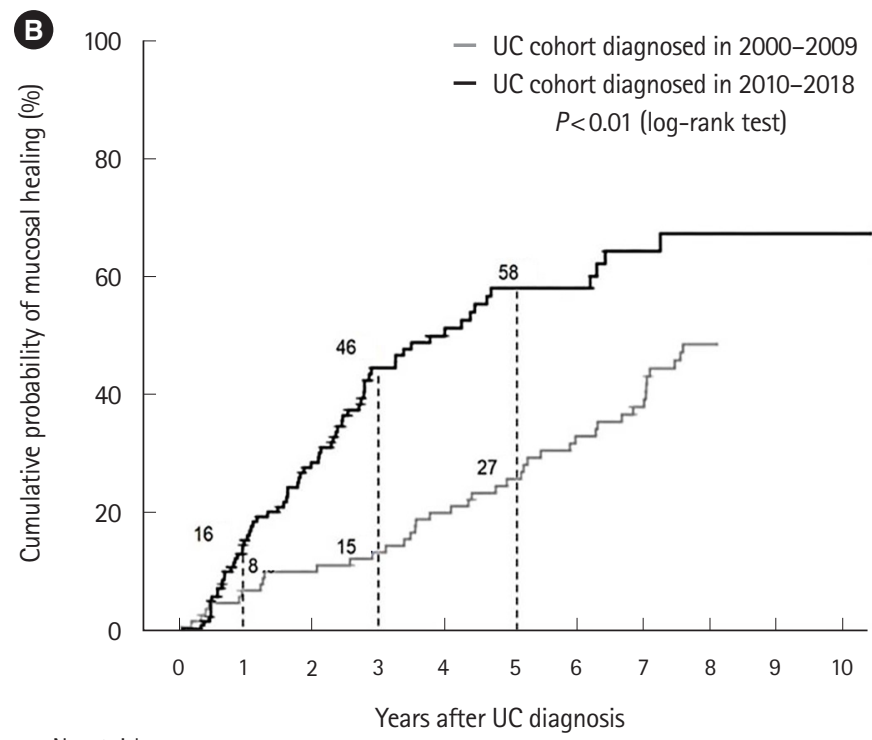

No. at risk

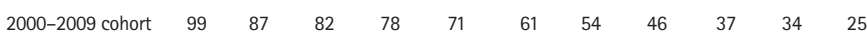
$\begin{array}{llllllllll}2010-2018 \text { cohort } & 153 & 109 & 84 & 51 & 37 & 29 & 23 & 11 & 7\end{array}$

Fig. 1. Cumulative probability of thiopurine use (A) and endoscopic mucosal healing (B) stratified by the calendar year of diagnosis. UC, ulcerative colitis.

Table 2. Univariate and Multivariate Analysis for Risk of Achieving Endoscopic Healing in 252 Patients with UC Diagnosis between 2000 and 2018

\begin{tabular}{|c|c|c|c|c|}
\hline \multirow{2}{*}{ Endoscopic healing } & \multicolumn{2}{|c|}{ Univariate analysis } & \multicolumn{2}{|c|}{ Multivariate analysis } \\
\hline & $\mathrm{HR}(95 \% \mathrm{Cl})$ & $P$-value & $\mathrm{HR}(95 \% \mathrm{Cl})$ & $P$-value \\
\hline Male sex & $0.65(0.46-0.94)$ & 0.02 & $0.71(0.50-0.97)$ & 0.07 \\
\hline Age at diagnosis $(<40 \mathrm{yr})$ & $0.71(0.49-1.01)$ & 0.06 & $0.69(0.48-1.00)$ & 0.05 \\
\hline BMI (per 1 unit increase) & $1.03(0.98-1.08)$ & 0.26 & - & - \\
\hline Ever/current smoker & $1.07(0.61-1.86)$ & 0.82 & - & - \\
\hline Extensive colitis & $0.76(0.53-1.09)$ & 0.10 & $0.82(0.57-1.17)$ & 0.28 \\
\hline Extraintestinal manifestation & $0.73(0.40-1.33)$ & 0.30 & - & - \\
\hline Calendar period of diagnosis in 2000-2009 & $0.55(0.38-0.79)$ & $<0.01$ & $0.54(0.37-0.79)$ & $<0.01$ \\
\hline
\end{tabular}

$\mathrm{UC}$, ulcerative colitis; $\mathrm{HR}$, hazard ratio; $\mathrm{Cl}$, confidence interval; $\mathrm{BMI}$, body mass index. 

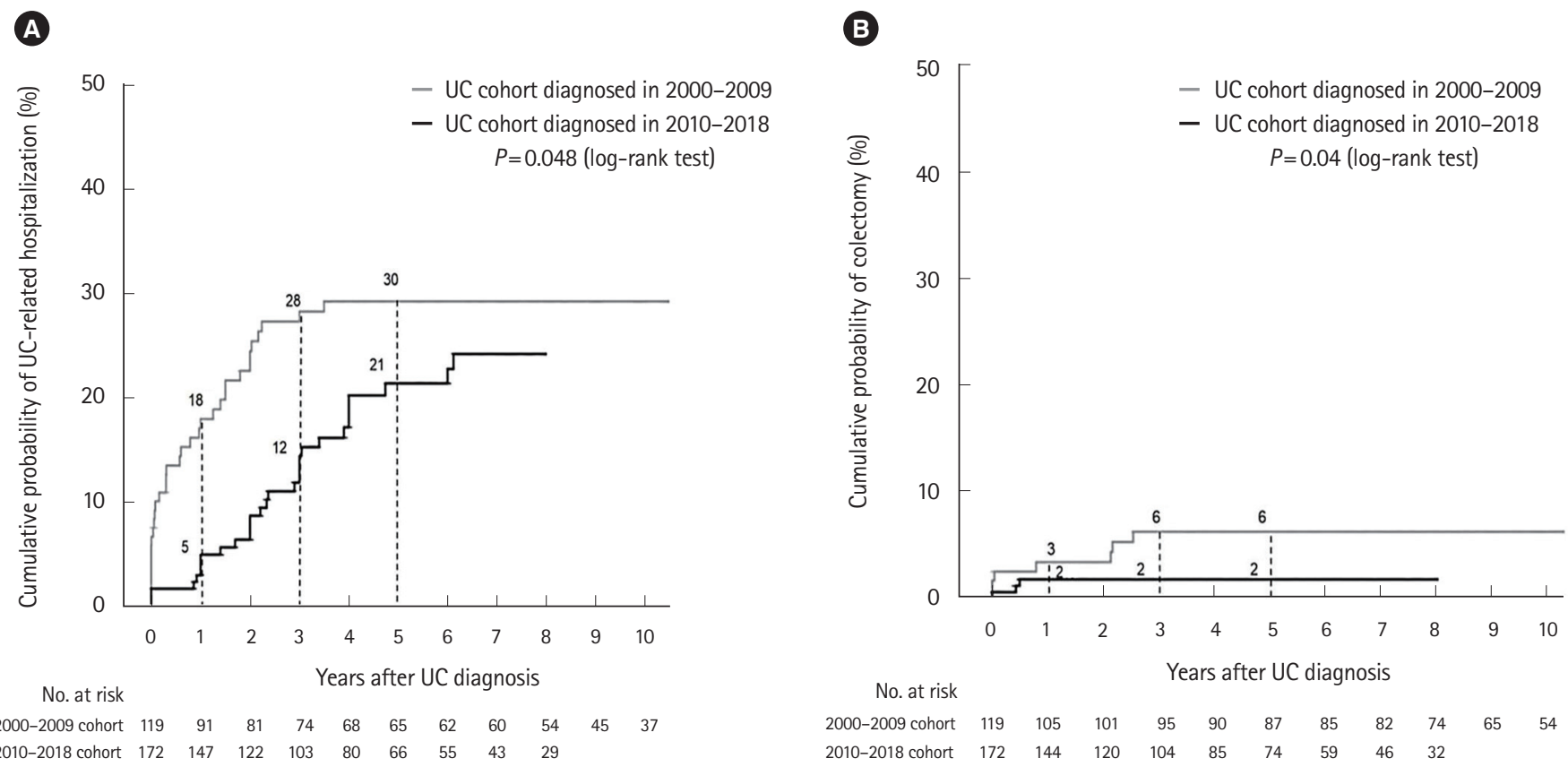

Fig. 2. Cumulative probability of requiring ulcerative colitis (UC)-related hospitalization (A) and colectomy (B) stratified by the calendar year of diagnosis.

years after diagnosis between the 2000-2009 cohort and the 2010-2018 cohort $(P<0.01)$ (Fig. 1B). Table 2 shows the Cox proportional hazards analysis models for the association of baseline factors and the risk of achieving endoscopic healing. In multivariate analysis, a decreased risk of achieving endoscopic healing was observed in patients with age $<40$ years at diagnosis (adjusted HR [aHR], 0.69; 95\% CI, 0.48-1.00) and those with UC diagnosis in 2000-2009 (aHR, 0.54; 95\% CI, $0.37-0.79)$.

\section{Hospitalization}

Among 291 patients with UC, 62 patients (21\%) required at least one hospitalization at some point during the disease course (33 patients from the 2000-2009 cohort and 29 patients from the 2010-2018 cohort). There was a significant decrease in the rate of UC-related hospitalization in the 20102018 cohort when compared with those in the 2000-2009 cohort $(P<0.05)$. The cumulative probability of requiring UC-related hospitalization was $18 \%$ versus $5 \%$ at 1 year, $28 \%$ versus $12 \%$ at 3 years, and $30 \%$ versus $21 \%$ at 5 years in the 2000 2009 and 2010-2018 cohorts $(P=0.048)$ (Fig. 2A). Table 3 shows the Cox proportional hazards analysis models for the association of baseline factors and the requirement of UC-related hospitalization. In multivariate analysis, patients with extensive colitis (aHR, 2.82; 95\% CI, 1.66-4.82) and those with
UC diagnosis in 2000-2009 (aHR, 1.70; 95\% CI, 1.03-2.81) were significantly associated with developing UC-related hospitalization.

\section{Colectomy}

Sixteen of 291 patients with UC underwent colectomy at maximal follow-up of the study (11 from the 2000-2009 cohort due to medical failure $[\mathrm{n}=8]$, superimposed severe cytomegalovirus colitis [ $\mathrm{n}=2]$, high-grade dysplasia of colon $[\mathrm{n}=1]$ and 5 from the 2010-2018 cohort due to medical failure $[\mathrm{n}=5]$ ). The rate of colectomy in the 2010-2018 cohort significantly decreased when compared with the 2000-2009 cohort $(P<0.05)$. The cumulative probability for colectomy at 5 years after diagnosis was $6 \%$ in the 2000-2009 cohort and $2 \%$ in the 2010 2018 cohort $(P=0.04)$ (Fig. 2B). In multivariate analysis, although not statistically significant, the risk of colectomy was also found in patients with extensive colitis (aHR, 1.96; 95\% CI, 0.69-5.51) and those with UC diagnosis in 2000-2009 (aHR, 2.81; 95\% CI, 0.92-8.61) (Table 3).

\section{Colorectal Cancer and UC-Related Death}

None of patients had colorectal cancer. One patient maintaining with thiopurine in the 2000-2009 cohort deceased from severe bacterial pneumonia. No death occurred in the 2010 2018 cohort. 
Table 3. Univariate and Multivariate Analysis for Risk of UC-Related Hospitalization and Colectomy in 291 Patients with UC Diagnosis between 2000 and 2018

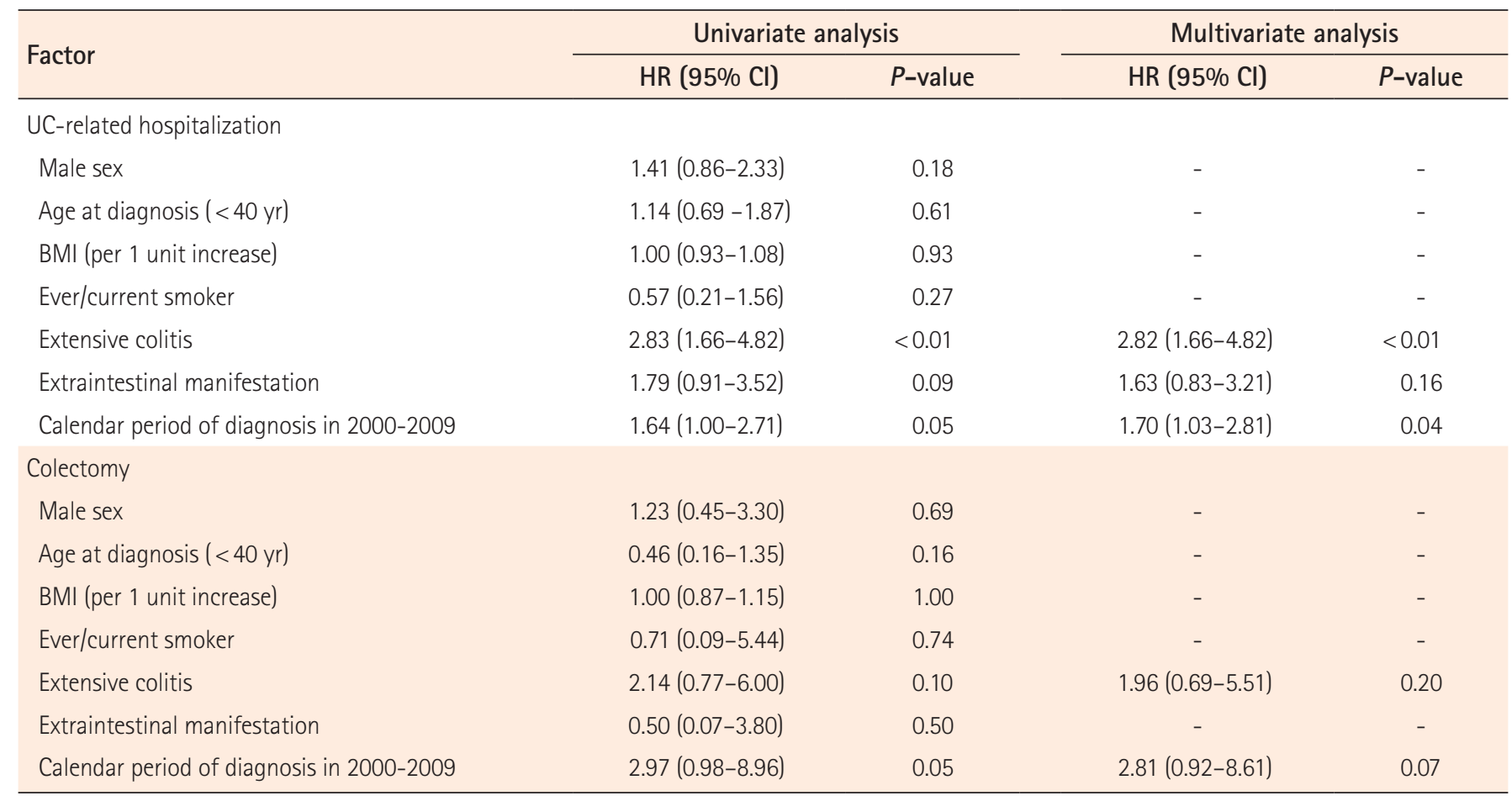

$\mathrm{UC}$, ulcerative colitis; $\mathrm{HR}$, hazard ratio; $\mathrm{Cl}$, confidence interval; $\mathrm{BMI}$, body mass index.

\section{DISCUSSION}

In the present study, we observed that the patient's characteristics and the disease extension at diagnosis of UC remained stable over time. There was a less aggressive disease course of UC. Approximately, $60 \%$ of patients had E1/E2 and about $14 \%$ of those patients developed E3 on follow-up. Although twothirds of patients experienced active disease that required systemic corticosteroid during their disease course, approximately the overall 5-year cumulative risk of requiring hospitalization was $25 \%$ and the overall 5 -year cumulative risk of colectomy was $5 \%$. These rates have been declined over time that corresponds with an increase in the use of thiopurine (63\%), but not biologic use (1\%).

There was no sex difference in our study population. Our findings are in line with a pooled analysis of incidence from Western studies. ${ }^{6}$ The disease locations of UC in our study were generally similar to those in the Western, ${ }^{3,7}$ Australian, ${ }^{4}$ and Asian populations. ${ }^{4}$ However, the overall rate of progression to extensive colitis was $14 \%$, which was comparable to the Asia population $(12 \%)^{8}$ but less than that in the Copenhagen study (28\%). ${ }^{9}$ In addition, the overall rate of requiring corticosteroid use in our study was approximately $63 \%$, which was relatively less than that in the Sweden study (71\%). ${ }^{7}$

The paradigm shifts in UC management such as early initiation of thiopurine and treatment target for endoscopic remission during the two decades were observed. We found a significantly increased proportion of patients with UC diagnosed in the 2010s who received more combinations of oral and topical mesalamine. The shorter time to initiate thiopurine after diagnosis and the proportion of thiopurine users has significantly increased during two decades. In the 2000s, approximately $20 \%$ of patients with UC used thiopurine in the first year after diagnosis and increased to $30 \%$ of patients with UC in the 2010s. This is in accordance with Dutch study, where there was a 2- to 3 -fold increased thiopurine user from $8 \%$ in 1991-1997 to $23 \%$ in $1998-2006 .{ }^{10}$ For biologic agents, we did not find the changes in the use of biologic agent in our population. The overall biologic agent use was only $1 \%$ for both the 2000 s and 2010s in our population. In contrast, an increase in the first year use of biologic agent (5\%-10\%) was found more in the West population. ${ }^{9-11}$ A Canadian cohort in 2014 reported that the cumulative incidence of biologic agent use within 5 years of diagnosis was $7.8 \%{ }^{12}$ This may explain by the disease course in our population, which was less severity, and then was able to control inflammation with conventional 
treatment. Regarding endoscopic remission, we demonstrated the significantly increased proportions of mucosal healing during two decades. The cumulative probability for achieving endoscopic healing within 3 years of the disease course was $15 \%$ in the 2000 s and increased to $46 \%$ in the 2010 s.

We found that the evolution of UC management, including the use of a combination of oral and topical mesalamine, ${ }^{13}$ early use of thiopurine ${ }^{14}$ and treat-to-target strategy, ${ }^{15}$ may play role on the changes long-term disease outcomes. The UC-related hospitalization and colectomy rates in our population were lower than those in the West. ${ }^{3}$ We also demonstrated a reduction rate of UC-related hospitalization and colectomy over the past two decades. Between the 2000s and 2010s, the 5-year cumulative probabilities for UC-related hospitalization decreased from $30 \%$ to $21 \%$ and for colectomy decreased from $6 \%$ to $2 \%$. A recent systemic review of Western studies showed that the 5 -year cumulative probability of requiring UC-related hospitalization was $29 \%$ to $54 \%$, and the colectomy rate at 5 years of disease was $10 \%{ }^{3}$

There have been no population-based data about the change of UC incidence over time from Thailand, and other countries in Southeast Asia. A hospital-based registry study from Malaysia demonstrated the crude UC incidence in 1980s was 0.3 per 100,000 and increased to 0.9 per 100,000 in $2010-2018 .{ }^{16}$ Disease emergence in developing countries suggests that changing epidemiological trends are related to westernized lifestyle and industrialization. A population-based study from the AsiaPacific region revealed that a high-density population was more likely to develop UC. ${ }^{17}$ Thus, in the 21 st century, UC is becoming a burden disease across Asia. ${ }^{1}$ Our results may help a newly industrialized country with a low incidence of UC with further investigations regarding environmental determinants of UC development, planning for a local health care strategy to modify the disease course of UC, and providing comprehensive information about disease prognosis to a patient for making a treatment decision.

A strength of this study is that all patients were meticulously longitudinally reviewed by 2 experts rather than relying on administrative data. All incident patients were followed from disease onset until the end of the study period. However, this study has several limitations. First limitation is a retrospective nature of the study; any data not recorded in the medical records would have been missed. Second, this study is a hospital-based study. Therefore, our patients would have more severe disease than these from a population-based study. We estimated the natural history of UC would be less aggressive in the national level. Third, changes in disease extent and endoscopic healing may not be accurate. Endoscopic evaluation was not performed routinely for all patients. In our practice, we generally perform endoscopic assessment in patient with flare-up disease. For patients with active disease, we usually perform endoscopic assessment every 6-12 months. Fourth, we did not classify disease activity according to disease activity indexes such as Mayo scores. Because disease course of UC is dynamic from disease in remission to severe disease over time, adjustment for the activity of disease would have been complicated. Fifth, we did not perform the analysis on time to initiation treatment of mesalamine and systemic corticosteroids. Certain therapies, most notably mesalamine was prescribed as an initial treatment for mild to moderate disease activity whereas systemic corticosteroid was prescribed as an initial treatment for severe disease activity. However, our study allows for an assessment of ever used of combination oral and topical of mesalamine and ever used of systemic corticosteroid in UC patients. Last, the ethnic diversity in Bangkok was less than in the Asia region. Therefore, these results would limit the generalization to the country with more diverse ethnicities.

In conclusion, the disease course of UC in Thailand was less aggressive in term of the rates of systemic corticosteroid use, biologic use, colectomy and UC-related hospitalization than in the West. Over the two decades, the rates of UC-related hospitalization and colectomy have been decreasing which were similar to the West.

\section{ADDITIONAL INFORMATION}

\section{Funding Source}

The authors received no financial support for the research, authorship, and/or publication of this article.

\section{Conflict of Interest}

No potential conflict of interest relevant to this article was reported.

\section{Author Contribution}

Conceptualization: Aniwan S, Limsrivilai J. Methodology: Aniwan S, Limsrivilai J. Formal analysis: Aniwan S. Data acquisition: Aniwan S, Limsrivilai J, Pongprasobchai S, Pausawasdi N, Prueksapanich P, Kongtub N, Rerknimitr R. Writing - original draft: Aniwan S. Writing - review and editing: Aniwan S, Limsrivilai J. Approval of final manuscript: all authors. 


\section{ORCID}

Aniwan S

Limsrivilai J

https://orcid.org/0000-0002-2730-2450

Pongprasobchai S

https://orcid.org/0000-0001-5867-0312

Pausawasdi N

Prueksapanich P

Kongtub N

Rerknimitr R https://orcid.org/0000-0002-1031-6549

https://orcid.org/0000-0002-3737-8555

https://orcid.org/0000-0001-5811-0120

https://orcid.org/0000-0003-1096-6522

https://orcid.org/0000-0001-6866-6886

\section{REFERENCES}

1. Ng SC, Shi HY, Hamidi N, et al. Worldwide incidence and prevalence of inflammatory bowel disease in the 21st century: a systematic review of population-based studies. Lancet 2018; 390:2769-2778.

2. Aniwan S, Harmsen WS, Tremaine WJ, Kane SV, Loftus EV Jr. Overall and cause-specific mortality of inflammatory bowel disease in Olmsted county, Minnesota, from 1970 through 2016. Mayo Clin Proc 2018;93:1415-1422.

3. Fumery M, Singh S, Dulai PS, Gower-Rousseau C, Peyrin-Biroulet L, Sandborn WJ. Natural history of adult ulcerative colitis in population-based cohorts: a systematic review. Clin Gastroenterol Hepatol 2018;16:343-356.

4. Ng SC, Tang W, Ching JY, et al. Incidence and phenotype of inflammatory bowel disease based on results from the Asiapacific Crohn's and colitis epidemiology study. Gastroenterology 2013;145:158-165.

5. Loftus EV Jr, Silverstein MD, Sandborn WJ, Tremaine WJ, Harmsen WS, Zinsmeister AR. Ulcerative colitis in Olmsted County, Minnesota, 1940-1993: incidence, prevalence, and survival. Gut 2000;46:336-343.

6. Shah SC, Khalili H, Gower-Rousseau C, et al. Sex-based differences in incidence of inflammatory bowel diseases-pooled analysis of population-based studies from western countries. Gastroenterology 2018;155:1079-1089.

7. Eriksson C, Cao Y, Rundquist S, et al. Changes in medical management and colectomy rates: a population-based cohort study on the epidemiology and natural history of ulcerative colitis in Örebro, Sweden, 1963-2010. Aliment Pharmacol Ther 2017;46:748-757.

8. Ng SC, Zeng Z, Niewiadomski O, et al. Early course of inflam-

matory bowel disease in a population-based inception cohort study from 8 countries in Asia and Australia. Gastroenterology 2016;150:86-95.

9. Vester-Andersen MK, Prosberg MV, Jess T, et al. Disease course and surgery rates in inflammatory bowel disease: a population-based, 7-year follow-up study in the era of immunomodulating therapy. Am J Gastroenterol 2014;109:705-714.

10. Jeuring SF, Bours PH, Zeegers MP, et al. Disease outcome of ulcerative colitis in an era of changing treatment strategies: results from the Dutch population-based IBDSL cohort. J Crohns Colitis 2015;9:837-845.

11. Vegh Z, Burisch J, Pedersen N, et al. Incidence and initial disease course of inflammatory bowel diseases in 2011 in Europe and Australia: results of the 2011 ECCO-EpiCom inception cohort. J Crohns Colitis 2014;8:1506-1515.

12. Targownik LE, Tennakoon A, Leung S, Lix LM, Singh H, Bernstein CN. Temporal trends in initiation of therapy with tumor necrosis factor antagonists for patients with inflammatory bowel disease: a population-based analysis. Clin Gastroenterol Hepatol 2017;15:1061-1070.

13. Ford AC, Khan KJ, Achkar JP, Moayyedi P. Efficacy of oral vs. topical, or combined oral and topical 5-aminosalicylates, in ulcerative colitis: systematic review and meta-analysis. Am J Gastroenterol 2012;107:167-176.

14. Eriksson C, Rundquist S, Cao Y, Montgomery S, Halfvarson J. Impact of thiopurines on the natural history and surgical outcome of ulcerative colitis: a cohort study. Gut 2019;68:623632.

15. Ungaro R, Colombel JF, Lissoos T, Peyrin-Biroulet L. A treatto-target update in ulcerative colitis: a systematic review. Am J Gastroenterol 2019;114:874-883.

16. Mokhtar NM, Nawawi KNM, Verasingam J, et al. A four-decade analysis of the incidence trends, sociodemographic and clinical characteristics of inflammatory bowel disease patients at single tertiary centre, Kuala Lumpur, Malaysia. BMC Public Health 2019;19:550.

17. Ng SC, Kaplan GG, Tang W, et al. Population density and risk of inflammatory bowel disease: a prospective populationbased study in 13 countries or regions in Asia-Pacific. Am J Gastroenterol 2019;114:107-115. 